\title{
Application of Reservoir-Predicting Technique to Thin Sandstones in Coal-Bearing Strata in Block K
}

\author{
Liu Leisong, Chen Zhigang ${ }^{*}$, Chen Jie, Ma Hui, Sun Xing, Wang Yuzhu, Han Yuchun \\ Geological Research Center, Bureau of Geophysical Prospect, Zhuozhou, China
}

\section{Email address:}

chenzhigang@cnpc.com.cn (Chen Zhigang)

${ }^{*}$ Corresponding author

\section{To cite this article:}

Liu Leisong, Chen Zhigang, Chen Jie, Ma Hui, Sun Xing, Wang Yuzhu, Han Yuchun. Application of Reservoir-Predicting Technique to Thin Sandstones in Coal-Bearing Strata in Block K. International Journal of Oil, Gas and Coal Engineering. Vol. 6, No. 5, 2018, pp. 88-95. doi: 10.11648/j.ogce.20180605.12

Received: May 30, 2018; Accepted: August 9, 2018; Published: September 10, 2018

\begin{abstract}
In Block K of South Turgay Basin in central Kazakhstan, the development of target, Aibalin, is controlled by the boundary of graben (especially rift-type stratigraphy-lithology assemblage). The Aibalin Fm is mainly developed with delta and lakeshore swamp facies, and composed of grey sandstone, siltstone, shale and coal-bearing strata, with extensive carbonized vegetal debris. Moreover, it contains thin and horizontally-variable reservoirs. Coal beds affect seismic survey greatly. Because of the influence of tuning effect in seismic data, thin sandstone reservoir distribution and physical properties cannot be reflected accurately in seismic data. Meanwhile, thin sandstone reservoir cannot be effectively predicted through seismic-based conventional inversion methods and processes. In this paper, a new prediction process for thin sandstone reservoir in this block is proposed, contributing to the effective prediction of thin sandstone reservoir distribution and physical properties. Firstly, sensitive parameters for lithology interpretation are defined and lithology interpretation template was established, through comprehensive analysis of drilling, logging and seismic data. Secondly, seismic wave impedance Bayes inversion genetic algorithm and cloud transform gamma attribute prediction technique are used to derive wave impedance and gamma data volume. Finally, the wave impedance and gamma data volume are combined with lithology interpretation template to predict the physical properties of the reservoirs.
\end{abstract}

Keywords: Coal Measure Strata, Sensitive Parameter, Genetic Method, Cloud Transform

\section{Introduction}

In Block K of South Turgay Basin in central Kazakhstan [1], the development of target, Aibalin, is controlled by the boundary of graben (especially rift-type stratigraphy-lithology assemblage). The Aibalin Fm is mainly developed with delta and lakeshore swamp facies, and composed of grey sandstone, siltstone, mudstone and coal-bearing strata, with extensive carbonized vegetal debris. Moreover, it contains thin and horizontally-variable reservoirs. Coal beds affect seismic survey greatly. Drilling data have proved that the thickness of Aibalin varies from 0 (uplift between grabens) to over $550 \mathrm{~m}$, or even $1300 \mathrm{~m}$ (graben axis). Aibalin is distributed inside graben-synclinorium [2], and deposited over the underlying Sajimubai Fm in unconformity form. The interval of interest is a set of lakeshore swamp facies coal-bearing sandstone and shale strata, with material source from the south to the north. Reservoirs in the target are thin and variable horizontally. Figure 1 is single-bed sandstone and coal bed distribution chart, on which the $\mathrm{X}$-axis is the single-bed thickness and the $\mathrm{Y}$-axis is frequency number. It can be seen that beds with thickness of $0.5 \mathrm{~m}$ and $1 \mathrm{~m}$ are more than those with other thicknesses. The accurate prediction of the distribution for these thin sandstone reservoirs is of great significance for the increment of reserves and production in Block $\mathrm{K}$.

Since its advent, the wave impedance inversion of post stack seismic data has been widely used in oil and gas exploration and development. Among all these inversion methods, the one based on convolution model has been used even widely due to its simple algorithm and insensitivity to seismic noise. This kind of inversion mainly includes direct inversion based on the deconvolution (such as the recursive inversion [3], trace integral inversion [4], etc.), model 
inversion (such as generalized linear inversion [5], generalized nonlinear inversion [6], logging constrained inversion [7], etc.), and perfect nonlinear inversion (including genetic algorithm [8], simulated annealing [9], artificial neural network [10], chaotic inversion [11], etc.). Being free from the constrain of restrictive assumptions of the search space and having no request on the continuity and conductivity, among all these perfect nonlinear inversion methods, the genetic algorithm inversion, a newly developed optimization algorithm simulating natural biological evolution process and mechanism based on Bayes formula [12], has become popular in the reservoir prediction in the field of oil and gas exploration.

In this study, seismic wave impedance Bayes inversion genetic algorithm and cloud transform [13] gamma attribute prediction method were adopted to predict the sandstone reservoirs in coal-bearing strata in Block $\mathrm{K}$. The results demonstrate that the sandstone development area and spatial distribution law are predicted accurately, thus providing reliable technical support for the increment of reserves and production in this block.

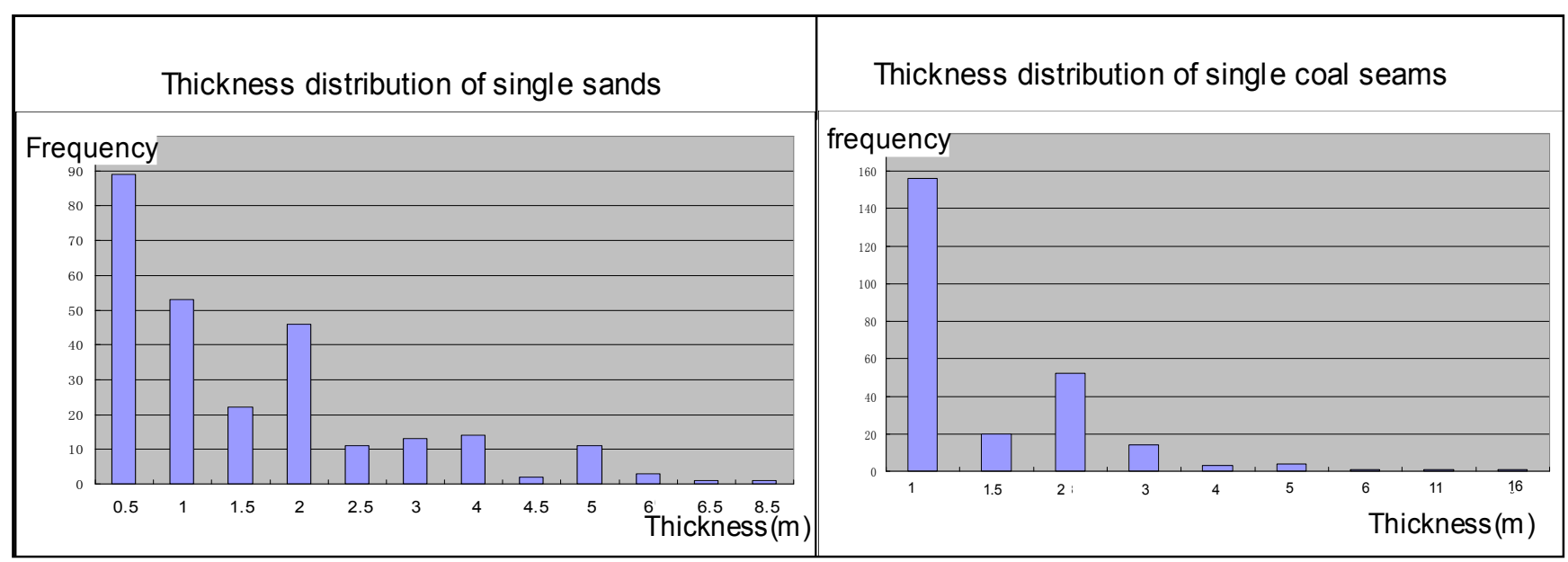

Figure 1. Statistics of single coal seams (left) and sands (right). The x-axis indicates single-layer thickness, and the y-axis indicates frequency.

\section{Method and Principles}

\subsection{Process of Wave-Impedance Random Genetic Inversion Algorithm}

Genetic algorithm (GA) is an optimization algorithm by simulating biological evolution process and mechanism in nature. The genetic inversion is based upon Bayes formula mathematically, i.e.:

$$
\frac{\mathrm{P}(\mathrm{E} \mid \mathrm{X}) \mathrm{P}(\mathrm{X} \mid \mathrm{H})}{\mathrm{P}(\mathrm{E} \mid \mathrm{H})}
$$

The Bayes formula can be written in form of likelihood $A$ and probability $\mathrm{O}$, namely, $\mathrm{O}(\mathrm{B} \mid \mathrm{A})=\mathrm{O}(\mathrm{B}) \mathrm{A}(\mathrm{B} \mid \mathrm{A})$, where $\mathrm{O}(\mathrm{B} \mid \mathrm{A})$ represents the posterior probability of event $\mathrm{B}$ in condition of event $\mathrm{A}$, and $\mathrm{O}(\mathrm{B})$ represents the prior probability of event $\mathrm{B}$ [14] Briefly, a "prior probability" is pre-estimated and then experiment result is introduced to determine whether the experiment strengthens or weakens the "prior probability"; in this way, a "posterior probability" closer to the reality is derived.

In inversion, the objective is to derive reservoir or such information as physical properties under the known logging, seismic and geological data. Given the geological data $\mathrm{H}$, logging, mud-logging and seismic data $\mathrm{E}$, and oil reservoir information $\mathrm{X}$, the Bayes formula can be written as:

$$
\mathrm{P}(\mathrm{X} \mid \mathrm{E}, \mathrm{H})=\frac{\mathrm{P}(\mathrm{E} \mid \mathrm{X}) \mathrm{P}(\mathrm{X} \mid \mathrm{H})}{\mathrm{P}(\mathrm{E} \mid \mathrm{H})}
$$

Inversion process is as follows:

1) Collect various geological data (logging, mud-logging and petrophysics [15]) and seismic data (pure-wave seismic data), and transform these information to prior probability distribution through analysis and processing.

2) With the collected data as constraint, establish initial model, with the maximum and minimum of model parameter controlled by prior information.

3) Calculate objective function or energy function of model parameter, modify model parameter randomly to generate new model, and calculate the prior probability of the model according to the transformed prior distribution.

4) In the first iteration, use the model for forward modeling, and then calculate the corresponding objective function or energy function.

5) Determine whether the objective function is adequate, modify if necessary, and generate new model.

6) Take the new model as model sample and store it in model sample space, and then calculate the likelihood function and posterior probability of the new model.

7) Repeat step (3) to step (6) on other samples.

8) Determine whether the conditions are satisfied, and output all sampling models and the corresponding posterior probability density functions.

9) Homogenize all posterior probability values, letting their sum equal to 1 ; then calculate the mean value and variance of model parameter, and output the inversion result. 


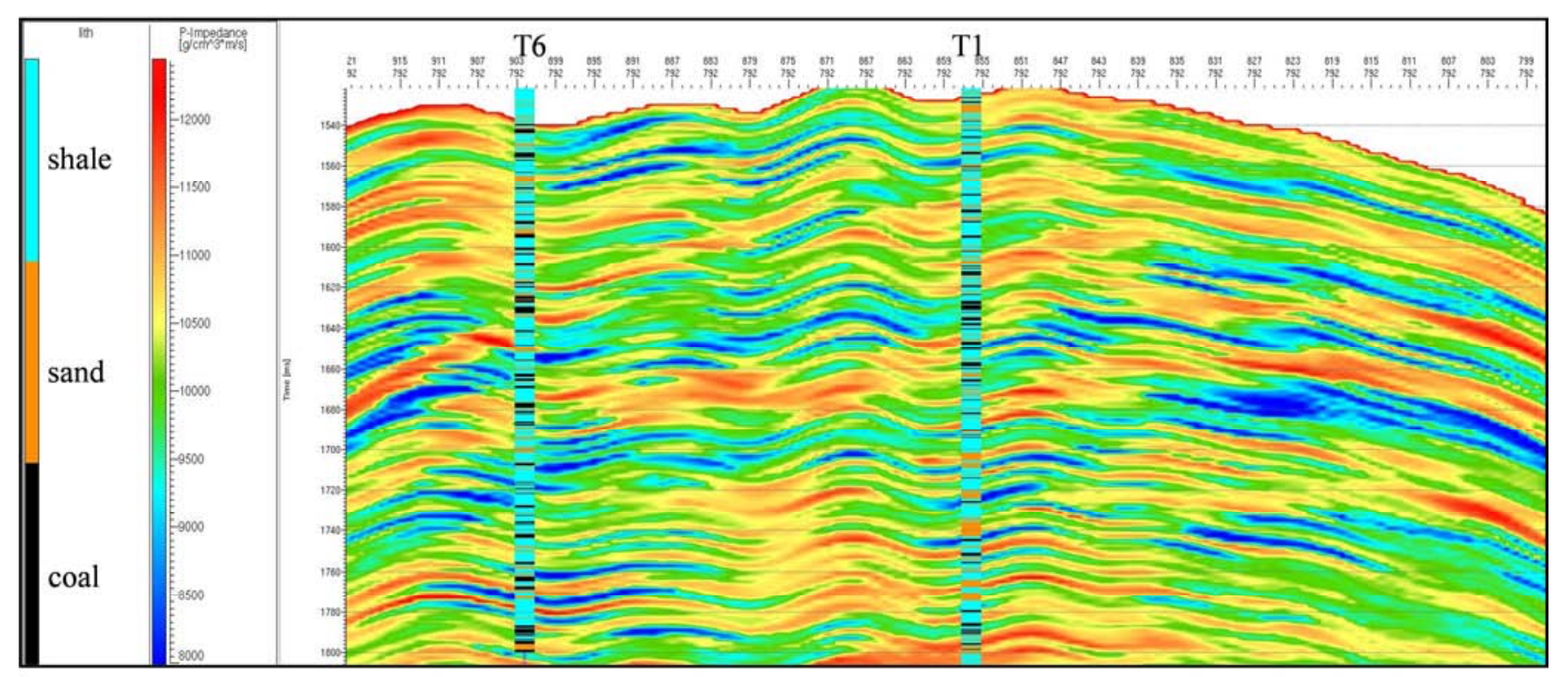

Figure 2. Genetic inversion result section.

Figure 2 shows the genetic wave-impedance random inversion section, where the density logging curve is presented by lithology data. The inversion method is of high resolution. The result on the section is consistent with the petrophysical analysis result previously, but impedance data volume cannot fully distinguish lithology.

\subsection{Cloud Transform}

Cloud transform is a nonlinear random simulation method. It transforms a variable to another variable through probability field simulation, subject to the complex nonlinear relation between the two variables.

Mathematically, cloud transform is defined as a transformation process from continuous numerical interval to discrete concept that given the frequency distribution function $f(X)$ of a certain data attribute $X$ in the universe, the stack of various clouds $\boldsymbol{C}\left(\mathrm{Ex}_{\mathrm{i}}, \mathrm{En}_{\mathrm{i}}, \mathrm{He}_{\mathrm{i}}\right)$ with different granularity is generated automatically according to the actual distribution of the frequency of $X$ attribute value. Each cloud represents a discrete and qualitative concept. Its mathematical expression is:

$$
f(\boldsymbol{X}) \rightarrow \sum_{i=1}^{n} a_{i} \boldsymbol{C}\left(E x_{i}, E n_{i}, H e_{i}\right)
$$

where $a_{\mathrm{i}}$ is the amplitude coefficient; $n$ is the number of the generated discrete concepts after transformation.

Cloud transform process includes detailed algorithm, realizing steps and decomposition method, etc.

1) Detailed algorithm
Input data set $D$ (original data, for example, input wave impedance data when converting wave impedance data to permeability data) and error threshold $\varepsilon$ (allowable error threshold) [16], and output $n$ concepts expressed by normal cloud model [17] through cloud transform algorithm.

2) Realizing steps

a. Calculate frequency distribution function $f(x)$ of data set $(\mathrm{f}(x)$ is a solution of $f(X))$.

b. Decompose $f(x)$ into the sum of $n$ normal functions. $n$ is determined by error threshold $\varepsilon$.

c. Calculate the expectation of cloud model according the decomposed normal functions.

d. Divide original data into $n$ data sets according the decomposed normal functions, and calculate the entropies and hyper entropies [18] of $n$ cloud models using reversed cloud algorithm in which certainty information is not necessary.

3) Decomposition method

a. Define the place where $f(x)$ wave peak locates as cloud expectation $E x_{i}$, estimate cloud model extropy $E n_{i}$ with expectation $E x_{i}$, and calculate cloud model data distribution function $f_{i}(x)$ to derive a fitting cloud.

b. Subtract the derived cloud model data distribution function $f_{i}(x)$ from it, and derive a new data distribution function $f^{\prime}(x)$.

According to the methods above, multiple cloud models can be derived. Figure 3 shows the gamma attribute section predicted by cloud transform, where density logging curve is presented by lithology information. Obviously, it is hard to fully distinguish lithology just through gamma section. 


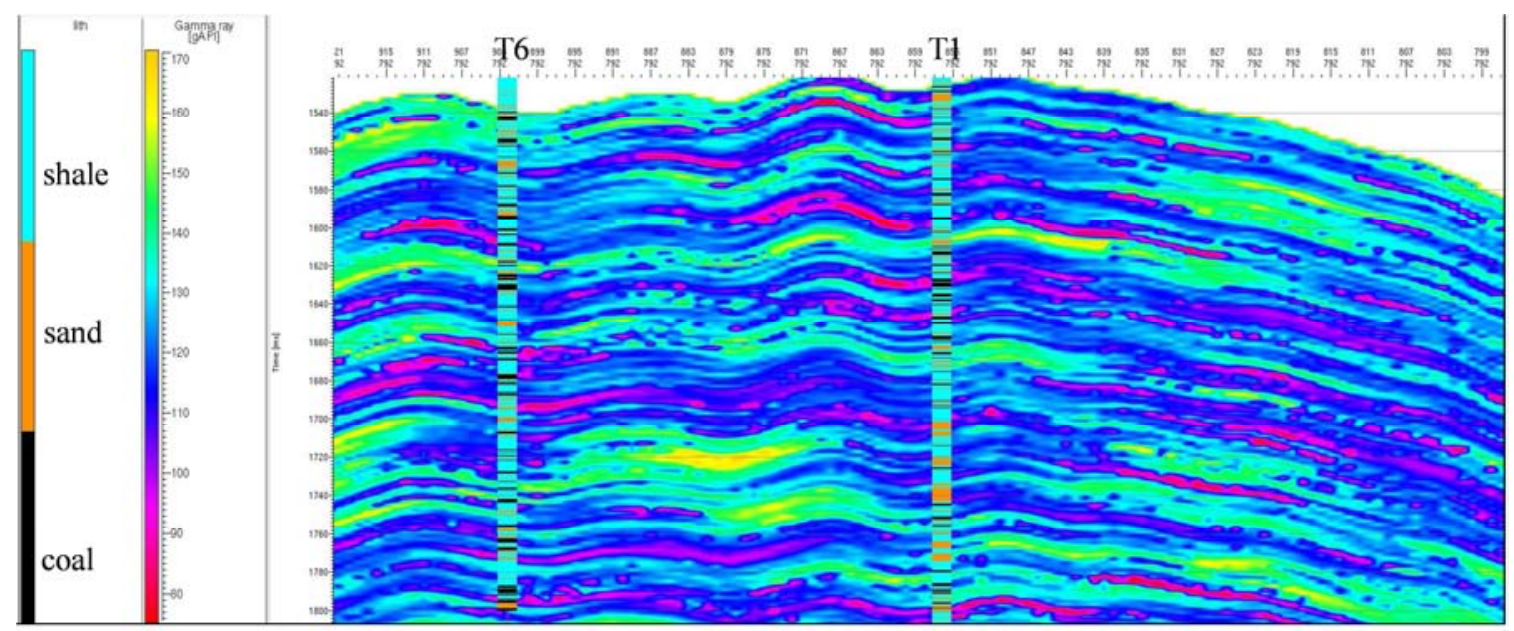

Figure 3. Predicted gamma attribute section.

\section{Thin Reservoir Prediction}

Considering the prediction difficulties in Block $\mathrm{K}$, a prediction process for thin sandstone reservoir is proposed in this paper. It can help realize the effective prediction of thin sandstone reservoir distribution and physical properties. Firstly, sensitive parameters for lithology interpretation are defined and lithology interpretation template was established, through comprehensive analysis of drilling, logging and seismic data. Secondly, seismic wave impedance Bayes inversion genetic algorithm and cloud transform gamma attribute prediction technique are used to derive wave impedance and gamma data volume. Finally, the wave impedance and gamma data volume are combined with lithology interpretation template to predict the physical properties of the reservoirs.

\subsection{Sensitive Parameter Analysis}

Considering the geological characteristics of Block $\mathrm{K}$, lithological sensitivity analysis is conducted to logging curves firstly. It can be seen from the wave impedance histogram in Figure $4 \mathrm{a}$ that coal-bed wave impedance values are lower than sandstone and shale wave impedance values, but the latters are superimposed and hard to be distinguished. It can be seen from the gamma histogram in Figure $4 b$ that sandstone and shale values are different, but coal-bed values vary from sandstone value range to shale value range. Figure 5 is the wave impedance and gamma cross plot, on which sandstone, shale and coal can be distinguished completely, thus the cross plot is taken as the theoretical basis of lithology prediction. Sandstone reservoir can be extracted through cross-plotting impedance and gamma. Meanwhile, as lithology interpretation template, the cross plot can help realize the quantitative interpretation for different lithologies in $3 \mathrm{D}$ attribute volume (sandstone: gamma<110API, wave impedance $\left.8000-14000 \mathrm{~g} \cdot \mathrm{cm}^{-3} \cdot \mathrm{m} \cdot \mathrm{s}^{-1}\right)$. According to the petrophysical characteristics above, it is necessary to combine wave impedance and gamma characteristics to distinguish sandstone, shale and coal, step by step. Wave impedance inversion and gamma attribute statistics prediction inversion are conducted; then, sandstone reservoir is predicted according to the cross plot of wave impedance and gamma attribute.

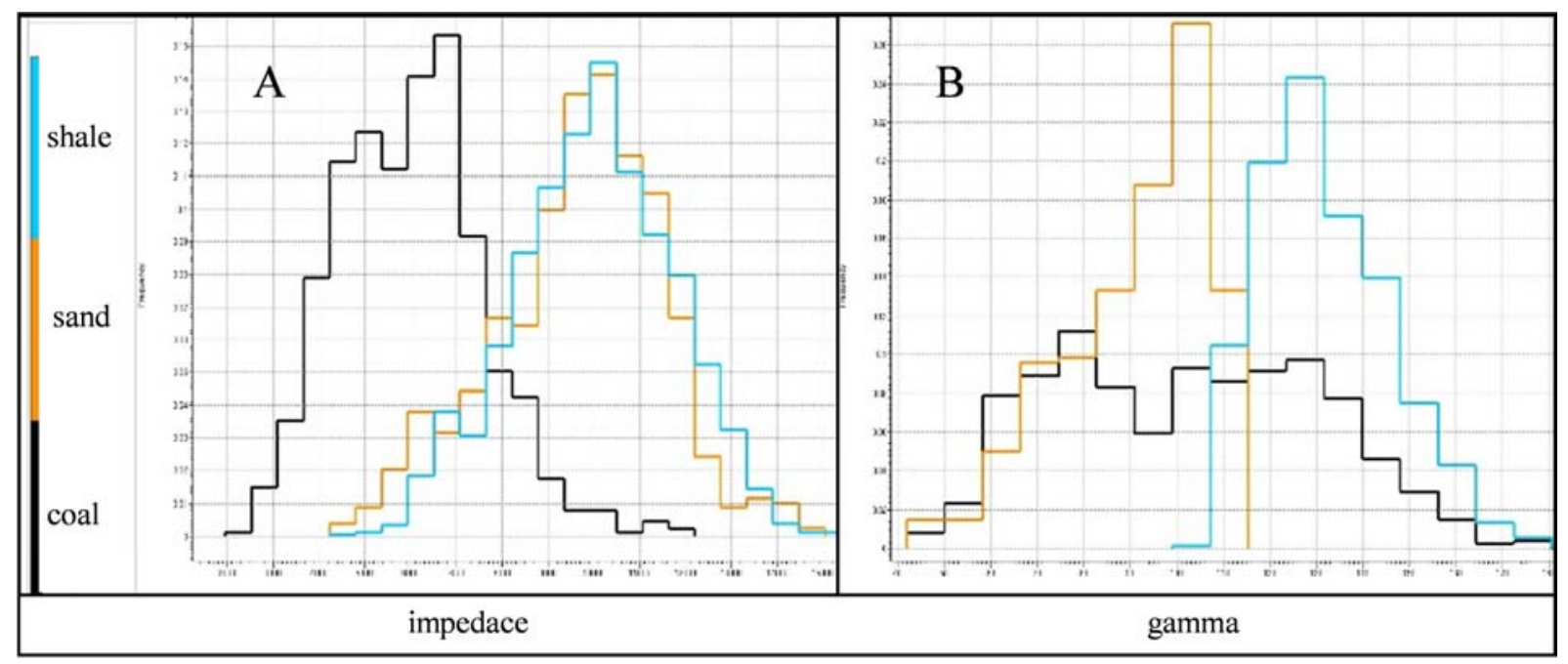

Figure 4. Wave impedance and gamma histograms. 


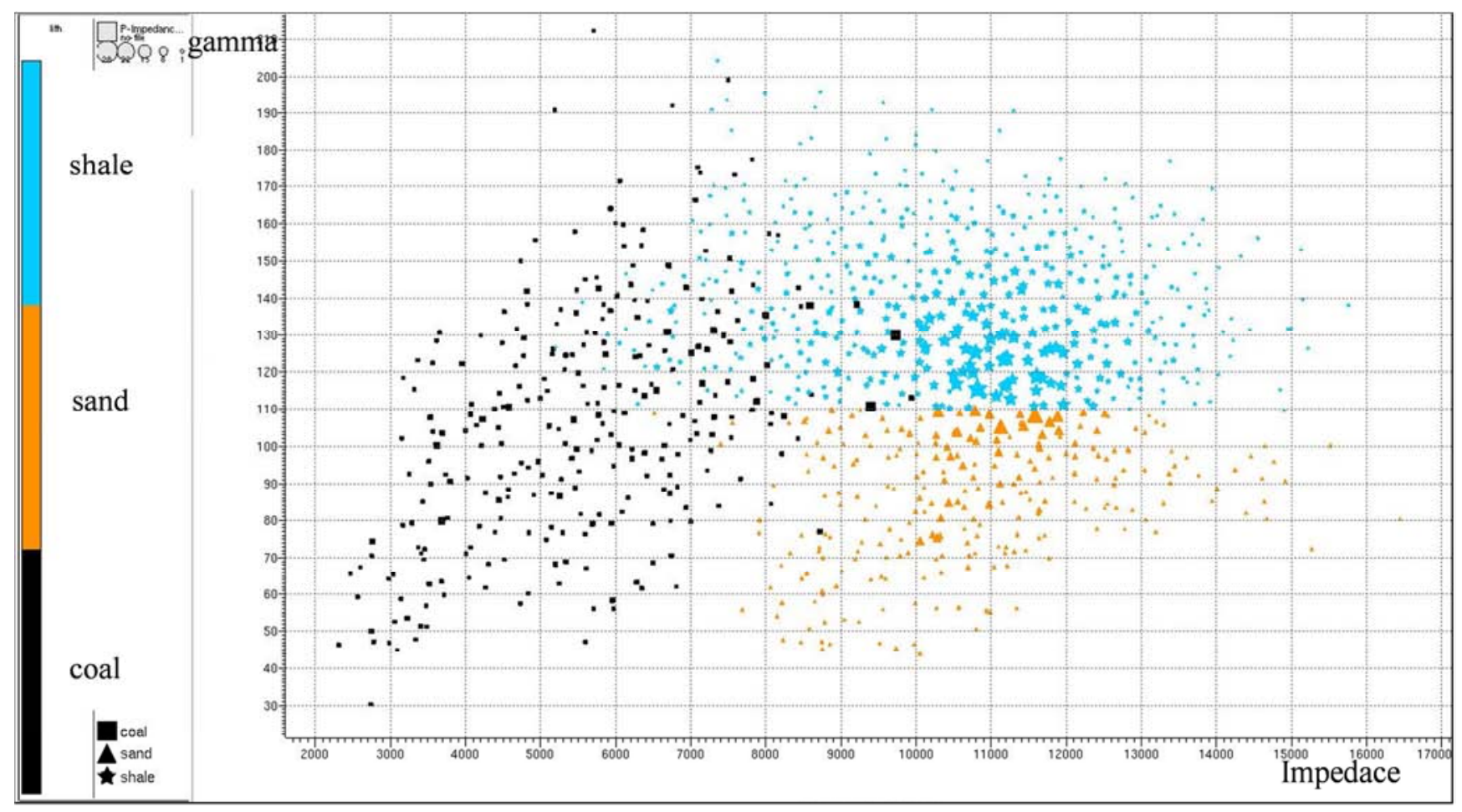

Figure 5. Wave impedance and gamma cross plot.

\subsection{Joint Application of Genetic Wave-Impedance Inversion and Cloud Transform}

As the first part of reservoir prediction, seismic data are analyzed and logging data are disposed. The 32-bit pure-wave seismic data with sampling interval of $1 \mathrm{~ms}$ are preferred; such data is information-abundant, enabling the prediction result to be closer to real geological situation.

Logging data are usually disposed and analyzed in two steps.

(1) Abnormal value elimination, scale normalization, depth matching and correction, and logging interpretation.

Logging interpretation involves lithology, porosity, permeability and oil saturation, etc. As for lithology interpretation (mud-logging and core data are necessary to be considered as references if allowable), it is better to avoid too many categories - two or three categories are adequate generally. Too many lithological categories will cause heavy calculation load. On the other hand, genetic inversion will goes into endless loop if isolate lithology exists. So, it is necessary to simplify the categorization if there are multiple lithologies in the block. As for porosity, permeability and saturation interpretation, it is better to have comprehensive original logging data, in which neutron, acoustic, density, deep and shallow lateral logging data are indispensable (the lack of these data will affect logging interpretation result), and LWD or oil production test data will be more helpful for the interpretation.

(2) Logging data analysis and interpretation to find the sensitive logging curves for lithology interpretation and hydrocarbon.

Acoustic time difference curve is indispensable in wave impedance inversion generally. As for logging curve sensitivity analysis, current logging curves are cross-plotted with acoustic time difference curve (or wave impedance curve). Lithology curve is selected as the color scale. If it is necessary to predict fluid, saturation curve should be chosen as color scale to realize sensitivity cross plot analysis. Poststack data are used for reservoir prediction. In lithology sensitivity analysis, the logging curve that can be used to effectively distinguish the lithology in cross-plotting with acoustic time difference curve (or wave impedance curve) should be figured out. Meanwhile, the lithology interpretation template is established.

Next, the genetic wave-impedance inversion and attribute volume cloud transform are conducted. If the cross plot of wave impedance and gamma is the best for distinguishing lithology in Block K, it is necessary to predict gamma attribute volume through cloud transform. In genetic wave impedance inversion, some aspects should be considered. First, proper probability distribution simulation function should be found when applying wave-impedance logging data probability distribution analysis. Second, the parameters of variogram [19] should be set through analyzing the spatial development and distribution of geological bodies according to regional geological analysis. Third, the seismic attribute should be controlled properly. When the quality of seismic data is poor, the control is as little as possible. When the quality of seismic data is fine, more control can be applied, but not exceed $30 \%$ generally.

In cloud transform process, the establishment of cloud model is important. The closer the established cloud model is to scatter crossing distribution configuration, the closer the cloud transform prediction result will be to real prediction attribute. 


\section{Application Effect}

The method proposed is applied to Block K. Through sensitive parameter analysis, it can be concluded that the cross plot of logging data wave impedance and gamma can help distinguish sandstone, shale and coal beds (Figure 5). Thus Figure 5 can be taken as the template for interpreting lithology in this block. The wave impedance data volume is derived through wave impedance inversion and the gamma volume is derived through cloud transform on the basis of wave impedance inversion. The two data volumes are used for 3D spatial cross-plotting. The human-computer interactive interpretation is conducted using lithology interpretation template (Figure 5). For example, in sandstone horizontal distribution interpretation, the area where wave impedance is larger than $8000 \mathrm{~g} \cdot \mathrm{cm}^{-3} \cdot \mathrm{m} \cdot \mathrm{s}^{-1}$ and gamma is smaller than 110API is selected, and the qualified time accumulation thickness in target in 3D space is extracted. The thickness distribution is the sandstone thickness distribution configuration of target.
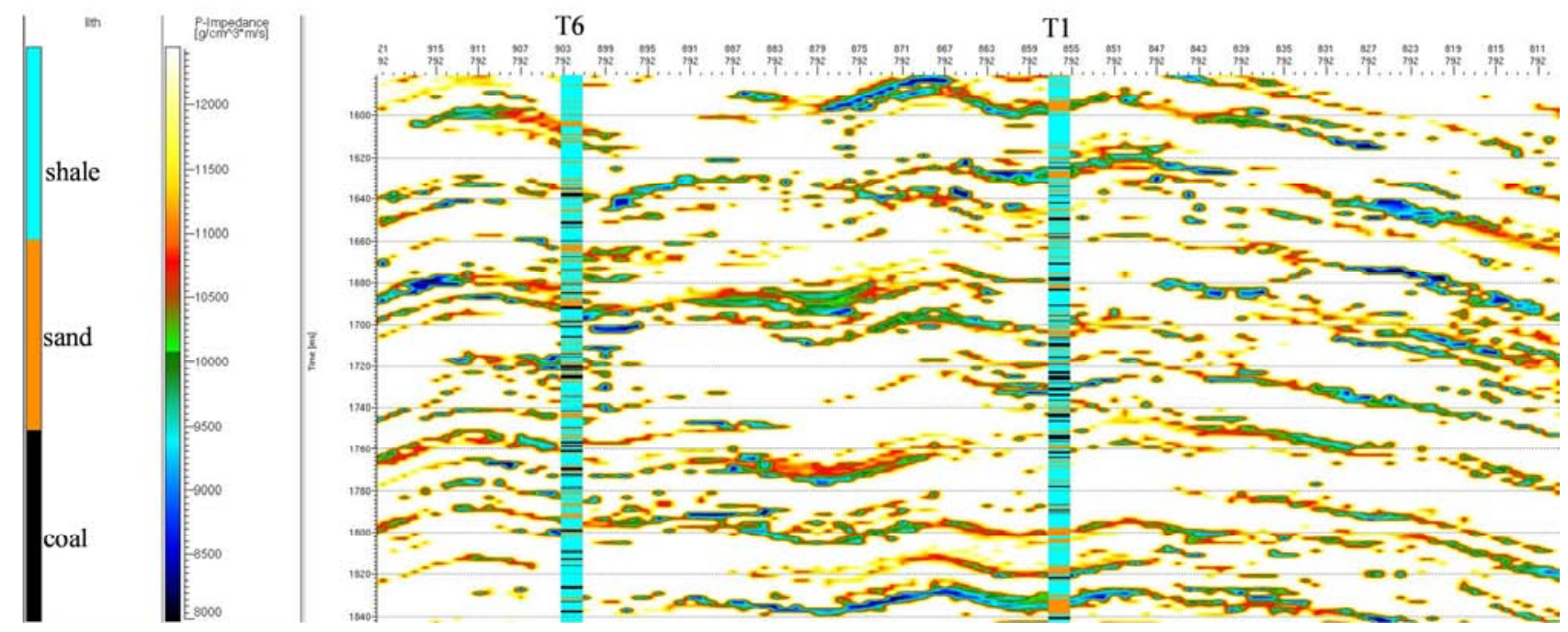

Figure 6. Cross-interpreted sandstone body section.

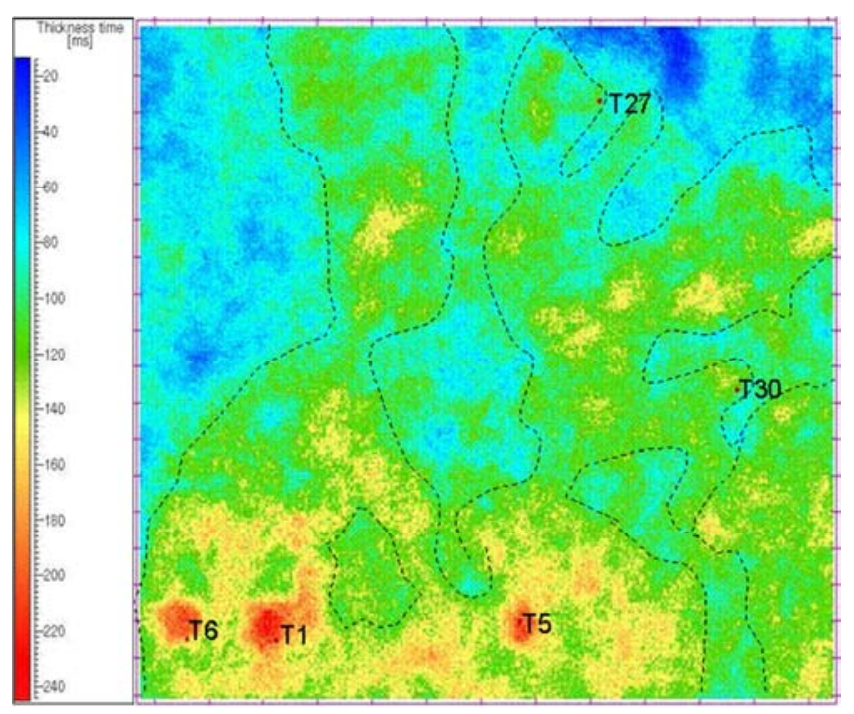

According to the prediction result (Figure 7), the sandstone is thick in the south and thin in the north, and it distributes in network, satisfying the geological law in this block.

Figure 8 shows the predicted sandstone reservoir thickness at well points and actual drilling data. The left side of Figure 8 is residual impedance section after eliminating coal and shale, where the information at well point is presented as lithology, and the locations marked with arrows are the corresponding points of predicted and actual drilling data. The right side of Figure 8 shows the bar chart comparing the prediction and the actual drilling data. It can be seen that sandstone thicker than 1 $\mathrm{ms}$ (equivalent to $2 \mathrm{~m}$ ) can be predicted accurately, and the sections with prediction error less than $1 \mathrm{~ms}$ are over $90 \%$ in the whole well section, reaching the requirement of reservoir prediction.

Figure 7. Sandstone thickness prediction result. 


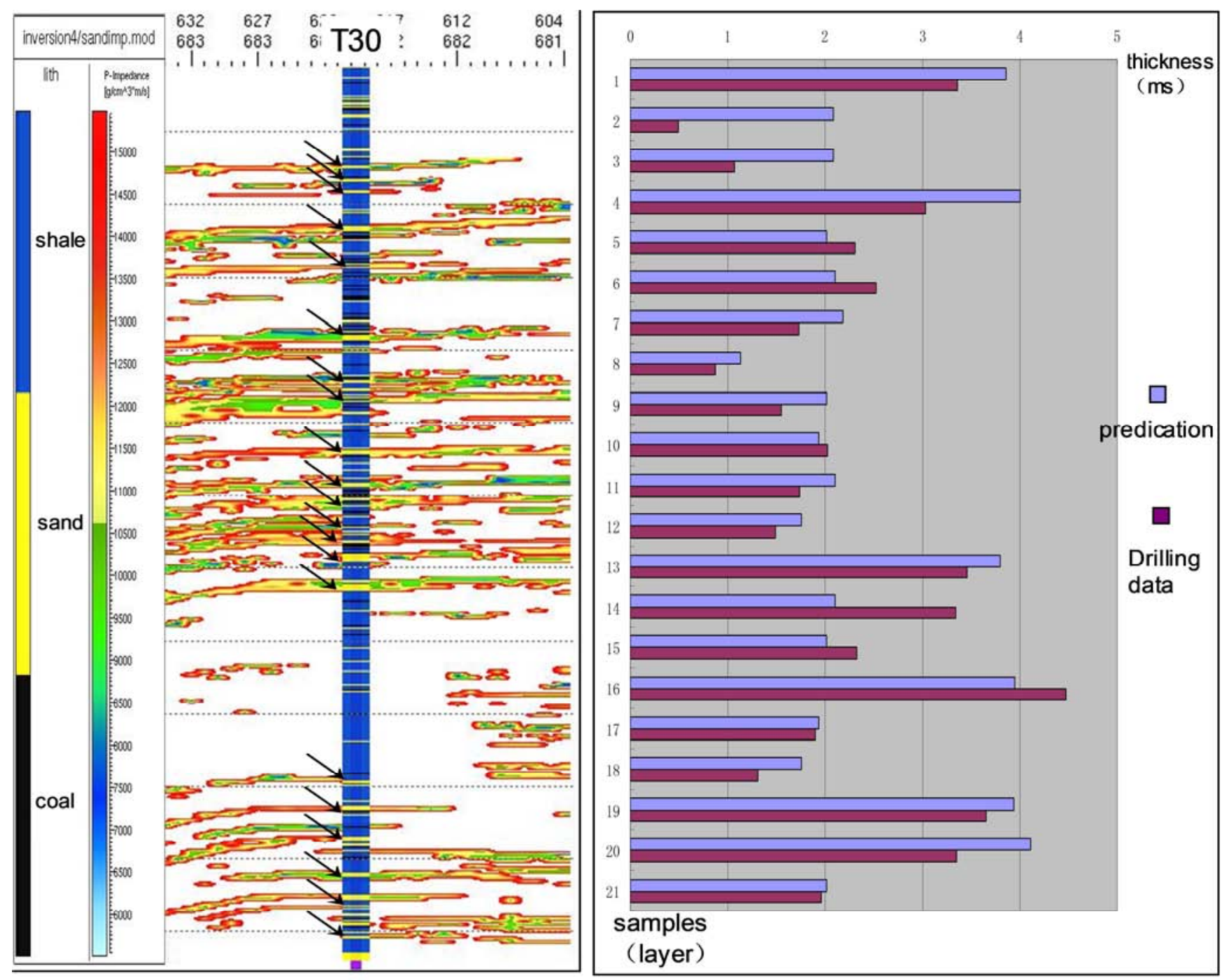

Figure 8. Comparison between prediction and drilling data of sandstone thickness at Well T30.

Note: The locations pointed by $\searrow$ are drilling and prediction comparison points. There are 21 sampling points totally. The right side shows the bar chart of the corresponding sampling points.

\section{Conclusions}

Through the comprehensive analysis on the application effect of thin sandstone reservoir prediction technology in coal-bearing strata in Block $\mathrm{K}$, the following conclusions can be derived:

(1) Compared with conventional inversion methods, the random seismic inversion based on genetic algorithm and cloud transform method are more suitable for the prediction of thin sandstone spatial distribution in coal-bearing strata in this block.

(2) The spatial distribution characteristics of sandstone derived by the random seismic inversion based on genetic algorithm and cloud transform method match actual drilling data well.

(3) The accurate analysis of reservoir sensitivity parameters based on the comprehensive analysis of drilling (logging), seismic and geological data is critical for reservoir prediction.

\section{References}

[1] Shi Juye, Fan Tailiang, et al. Several Typical Seismic Facies in South Turgay Basin and the Geological Meaning. Science Technology and Engineering, 2015, 15(Supplement 34): 133-138.

[2] Yan Geng, Kong Linghong. Lithological trap identification and description technology under sequence stratigraphy framework-Kazakhstan South Turgay Basin Example. Geophysical Prospecting, 2013, 51(Supplement 1): 139-145.

[3] Hu Meilan. Application of Recursive Inversion in STRATA. Petrochemical Industry Application, 2013, 32(Supplement 11): 53-55.

[4] Zhang Junhua, Hou Jing, et al. Theory Annotation and Application of Trace Integration Attribute in The Prediction of Thin Channel Sand Body. Progress in geophysics. 2018, 33(Supplement 1): 326-333. 
[5] Fang Yuan, Zhang Fengqi, et al. Generalized Linear Joint PP-PS Inversion Based on Two Constraints. Applied Geophysics. 2016, 13(Supplement 1): 103-115.

[6] Cheng Jianhua, ScottE. Parker, et al. A Second-Order Semi-Implict of Method for Bybrid Simulation. Journal of Computational Physics. 2013, Volume 245, 15 July:364-375.

[7] Du Zeyuan, Wu Guochen, et al. Full Waveform Inversion Based on Well Logging Data Constraint. OGP. 2017, 52(Supplement 6): 1184-1192.

[8] Lan Tian, Gui Zhixian, et al. Improved Particle Swarm Impedance Inversion. Fault-Block Oil \& field. 2016, 23(Supplement 2): 176-180.

[9] Qin Jingxin, Hao Tianyao, et al. The Density Interface Inversion Method of Improved Adaptive Simulated Annealing. Progress in Geophysics. 2014, 29(Supplement 5): 2060-2065.

[10] Li Juanjuan, Cui Ruofei, et al. Coalfield Seismic Inversion Using Probabilistic Neural Network. Progress in Geophysics. 2012, 27(Supplement 2): 715-721.

[11] Zhang Jin, An Zhenfang, et al. Elastic Impedance Inversion Based on Chaos and Colony Algorithm. Geophysical Prospecting for Petroleum. 2015, 54(Supplement 6): 716-723.

[12] Yin Bin, Hu Xiangyun. Overview of Nonlinear Inversion Using Bayesian Method. Progress in Geophysics. 2016, 31(Supplement 3): 1027-1032.
[13] Liu Leisong, Gao Jun, et al. Application of Cloud Transforms in Seismic Reservoir Prediction, SEG Houston 2013 Annual Meeting:2480-2484.

[14] Zhang Yucun, Xu Fei, et al. Noise Cancellation Algorithm Method Combinated Priori Probability with Curve Probability Threshold Segmentation. China Mechanical Engineering, 2017, 28(Supplement 8): 936-945.

[15] Xie Fei, Ding Wenlong, et al. Petrophysical Properties of Continental Shale Reservoir and The Influence Factors of Its Gas Contents. Science Technology and Engineering. 2017, 17(Supplement 5): 20-28.

[16] Zhu Qidan, Jin Liqiu, et al. Improved algorithm of minimal error threshold division method. Opto-Electronic Engineering, 2010, 37(Supplement 7): 107-113.

[17] Fu Bin, Li Daoguo, et al. Review and prospect on research of cloud model. Application Research of Computers, 2011, 28(Supplement 2): 420-426.

[18] Yang Zhixiao, Fan Yanfeng. Cloud mapping and membership cloud of mapping. Application Research of Computers, 2012, 29(Supplement 2): 553-556.

[19] Shu Xiao, Song Yongkang, et al. The application of fine variogram analysis in geological statistical modeling. Nei Jiang Science \& Technology, 2013(Supplement 10): 74-75. 